\title{
Qualitätsziele und Doku-Regeln für DMP COPD überarbeitet
}

\begin{abstract}
— Der Gemeinsame Bundesausschuss (G-BA) in Berlin hat die Anforderungen an das Disease-Management-Programm (DMP) zur Behandlung von Patienten mit chronisch obstruktiver Lungenerkrankung (COPD) aktualisiert. Das
\end{abstract}

Bundesgesundheitsministerium kann den Beschluss binnen zweier Monate beanstanden, ansonsten wird er am 1. Januar 2017 in Kraft treten. Die Vertragspartner auf Landesebene haben dann bis zum 1. Januar 2018 Zeit, ihre Verträge zum DMP COPD anzupassen.

\section{MMW-KOMMENTAR}

Mit dieser zweiten Überarbeitung seit der Erstfassung 2004 passt der G-BA die inhaltlichen Vorgaben an neue wissenschaftliche Erkenntnisse an. Im Fokus stehen dabei die Qualitätsziele sowie die Dokumentation. Die wichtigste Änderung ist die stärkere Gewichtung der Tabakentwöhnung. Neu aufgenommen wurde z. B. der Anspruch des Patienten auf den Zugang zu einem strukturierten, publizierten Entwöhnungsprogramm. Ferner sollen mindestens jährlich sämtliche Arznei-

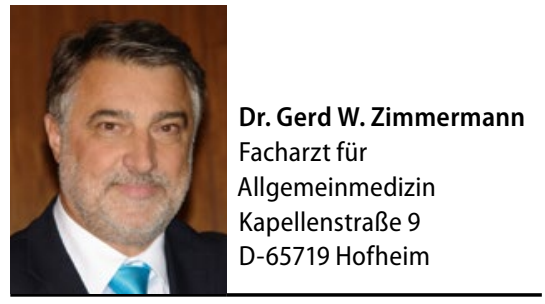

mittel einschließlich der Selbstmedikation strukturiert erfasst werden, um Therapieänderungen oder Dosisanpassungen frühzeitig vornehmen zu können - und dabei auch Nebenwirkungen und Interaktionen berücksichtigen zu können.

Es gibt neue Empfehlungen zu häufigen Komorbiditäten, insbesondere zur Osteoporose. Außerdem wurden Änderungen und Konkretisierungen bei der Diagnostik, der Medikation und den Überweisungsregeln vorgenommen. Um die Umsetzung der Empfehlungen zu unterstützen, wurden folgende neue Qualitätsziele aufgenommen: Teilnahme an einem Tabakentwöhnungsprogramm, jährliche Spirometrie, jährliche Einschätzung des individuellen Osteoporoserisikos, Vermeidung eines übermäßigen Einsatzes von inhalativen Steroiden und die Empfehlung zu körperlichem Training.

\section{Zahl der MVZ-Gründungen stagniert}

Laut KBV gab es am 31. Dezember 2015 bundesweit 2.156 Medizinische Versorgungszentren (MVZ). Sowohl prozentual als auch absolut nimmt der jährliche Zuwachs ab. Ende 2009 betrug er noch $20,6 \%$, im letzten Jahr waren es nur noch $4 \%$. Spitzenreiter bei den $\mathrm{Zu}$ lassungen sind die KV-Regionen Bayern, Niedersachsen, Hessen und Nordrhein. Jeweils 40\% der MVZ-Gründer sind Vertragsärzte und Krankenhäuser. Die bevorzugten Rechtsformen sind die Gesellschaft mit beschränkter Haftung $(\mathrm{GmbH})$ und die Gesellschaft bürgerlichen Rechts (GbR).

In den MVZ arbeiteten zum Stichtag 14.317 Ärzte. 91\% sind als angestellte
Ärzte tätig, davon 60\% in Teilzeit und 9\% als Vertragsärzte. Der Frauenanteil wurde nicht erhoben. Im Durchschnitt arbeiten in einem MVZ 6,6 Ärzte. Die Tendenz ist hier allerdings weiterhin steigend, sodass von einem Konzentrationsprozess ausgegangen werden kann. Hausärzte, fachärztlich tätige Internisten und Chirurgen sind die am häufigsten vertretenen Fachgruppen.

\section{MMW-KOMMENTAR}

Einige Details sind besonders interessant. So hat Bayern mit Abstand die meisten MVZÄrzte, nämlich 2.562 - aber den höchsten Anteil an der vertragsärztlichen Versorgung haben MVZ-Ärzte in den ostdeutschen Län- dern: 16,3\% in Thüringen, 13,4\% in SachsenAnhalt und 12,5\% in Sachsen. Die meisten MVZ sind hier in der Trägerschaft eines Krankenhauses. Lediglich die MVZ im Stadtstaat Hamburg kommen mit 15,9\% auf einen ähnlich hohen Versorgungsanteil, wobei sie hier zu vier Fünfteln von Vertragsärzten betrieben werden. Umgekehrt betrachtet werden in Thüringen durchschnittlich 20.939 Einwohner von einem MVZ versorgt, in Bayern 30.880 und in Baden-Württemberg, wo der Versorgungsanteil der MVZ-Ärzte bei 4,4\% liegt, 70.043 Einwohner.

Unverändert ist die räumliche Verteilung der Zentren: $46,6 \%$ stehen in Ballungsgebieten, $39,3 \%$ in mittelgroßen Regionen und nur $14,1 \%$ in ländlichen Regionen. 